\title{
Giant and multiple cardiac papillary fibroelastomas masquerading as acute cerebrovascular events
}

\author{
Cosmin Adrian TeOdoru'), Maria AleXANdra PredA ${ }^{2)}$, Veronica MădăLina Borugă ${ }^{3)}$,

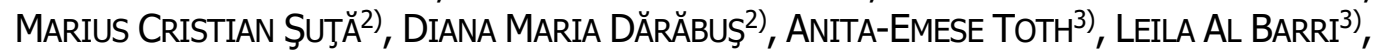

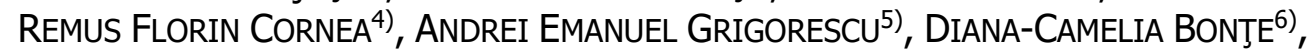 \\ SimONA STANCA ${ }^{7)}$, HOREA BOGdAN FEIER ${ }^{8)}$
}

\author{
1) Department of Ophthalmology, Victor Papilian Faculty of Medicine, Lucian Blaga University of Sibiu, Romania \\ 2) Department of Ophthalmology, Victor Babeş University of Medicine and Pharmacy, Timişoara, Romania \\ 3) PhD Student, Victor Babeş University of Medicine and Pharmacy, Timişoara, Romania \\ 4) Department of Pathology, Victor Babeş University of Medicine and Pharmacy, Timişoara, Romania \\ 5) Institute for Cardiovascular Diseases, Timişoara, Romania \\ 6) Department of Biochemistry, Victor Babeş University of Medicine and Pharmacy, Timişoara, Romania \\ 7) Department of Pediatrics, Carol Davila University of Medicine and Pharmacy, Bucharest, Romania \\ ${ }^{8)}$ Department of Cardiovascular Surgery, Victor Babeş University of Medicine and Pharmacy, Timişoara, Romania
}

\begin{abstract}
Cardiac papillary fibroelastomas are rare benign cardiac tumors. Their clinical significance results from their very high propensity for embolization. We present two such cases, which are rare regarding the size and multiplicity of these tumors.
\end{abstract}

Keywords: fibroelastoma, cardiac, papillary, cerebrovascular accident.

\section{a Introduction}

Cardiac papillary fibroelastomas (CPFs) are rare benign tumors, usually small and unique, arising most often from the valvular endothelium. Their prevalence varies from $0.02 \%$ to $0.08 \%$ in the medical literature $[1,2]$. Their clinical significance is due to the very high embolization propensity, which is usually in the cerebral circulation [ 1 , $3-5]$ but also in the coronary arteries [6,7]. While these tumors are mostly solitary, there have been reports of fibroelastomas in multiple locations [8-10], but this situation remains exceptional.

\section{Aim}

We present two cases in which both patients suffered neurological insults, and which stand out regarding the size and multiplicity of these tumors. These cases have been investigated and operated in the Departments of Ophthalmology and Cardiovascular Surgery, Timişoara, Romania, in 2016 and 2018, respectively.

\section{ㅁ Case presentations}

Due to the retrospective nature of the reports, written consent has been waived.

\section{Case No. 1}

A 62-year-old woman presented in the Emergency Department with sudden-onset aphasia. She was followed-up regularly for paroxysmal atrial fibrillation and her most recent transesophageal echogram revealed a left atrial appendage free from thrombi and normal functioning cardiac valves. She was treated with angiotensin-converting enzyme (ACE) inhibitors, diuretics, and vitamin K antagonists. Her most recent international normalized ratio (INR) was 2.11. A cerebral computed tomography (CT) diagnosed an ischemic cerebrovascular accident (CVA) in the anterior Broca area. The aphasia evolved into the expressive type and she was referred to Department of Cardiology for further check-up. A Holter exam revealed a normal sinus rhythm and no conduction disturbances. The carotid ultrasound (US) was normal. Transthoracic echography revealed flaky, small cardiac tumors at the level of the aortic valve, as well as severe, new-onset mitral regurgitation. A transesophageal US exam was performed, which showed no thrombus in the left atrial appendage and, at the level of the aortic valve, the presence of flaky, multiple small tumors (Figure 1). The surprise, however, was the presence of severe, eccentric mitral regurgitation (Figure 2), as well as the presence of cardiac tumors on the atrial side of the A2 segment of the mitral valve, of which the biggest was $7 \mathrm{~mm}$ in size (Figure 3). Her left atrial appendage was free from thrombi. Her coronary and carotid angiography was normal. She was admitted to Department of Surgery for curative treatment. The operation was performed by median sternotomy, under cardiopulmonary bypass. Exposure of the mitral valve through the interatrial groove revealed an eroded A2 leaflet of the mitral valve, as well as the

This is an open-access article distributed under the terms of a Creative Commons Attribution-NonCommercial-ShareAlike 4.0 International Public License, which permits unrestricted use, adaptation, distribution and reproduction in any medium, non-commercially, provided the new creations are licensed under identical terms as the original work and the original work is properly cited. 
presence of multiple small, friable cardiac tumors. It was resected en bloc and sent to pathology. The aortic valve was approached by a transverse aortotomy, which gained access to the aortic root. The inspection of the aortic valve revealed the presence of small, reddish, friable tumors on the non-coronary leaflet, close to the rightnon-coronary commissure (Figure 4). As the aortic valve was functioning normally, the tumors were shaved and

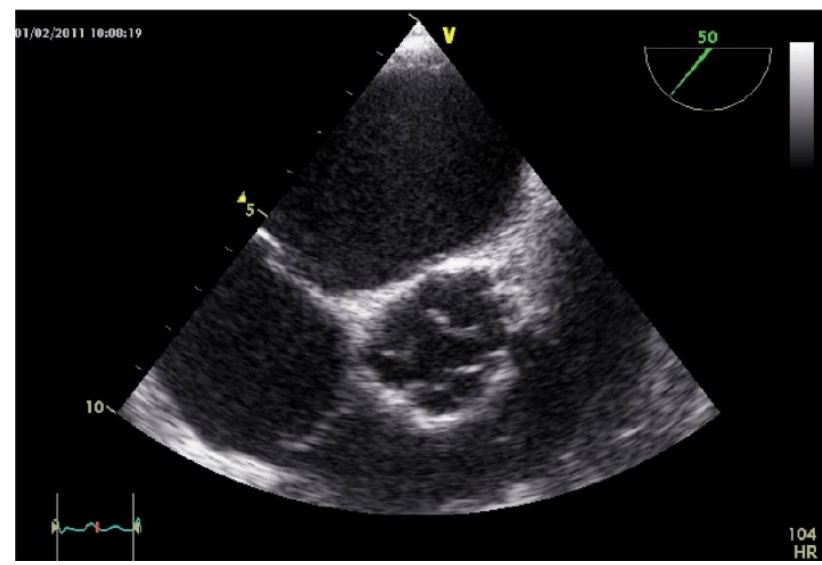

Figure 1 - Transesophageal short axis view of the aortic valve with multiple tumors present on the aortic surface of the leaflets.



Figure 3 - The presence of small, $7 \mathrm{~mm}$ in size tumors on the atrial aspect of the anterior mitral leaflet, at the A2 level.



Figure 5 - Hematoxylin-Eosin staining $(\times 40)$ of the resected specimen, showing an avascular collagen core surrounded by myxoid tissue. sent to Department of Pathology. The postoperative course was uneventful, and the patient was discharged on postoperative day 10 in sinus rhythm. The histopathological sections were stained with Hematoxylin-Eosin (HE) and the examination diagnosed multiple CPFs (Figures 5 and 6). The patient remains, five years later, in sinus rhythm with an expressive Broca aphasia.



Figure 2 - Transesophageal Doppler long-axis section of the mitral valve with a severe, eccentric mitral regurgitation.

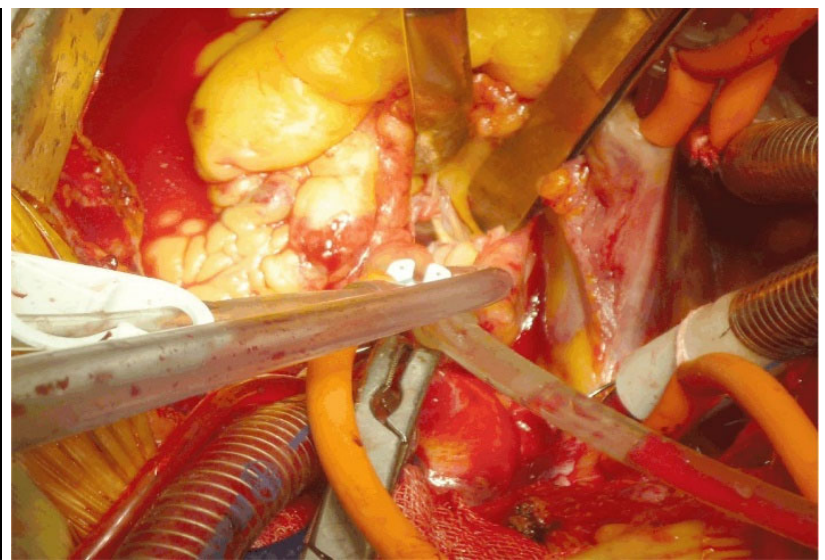

Figure 4 - Intraoperative view of the aortic valve, and a reddish, friable tumor on the non-coronary leaflet, close to the right-non-coronary commissure.

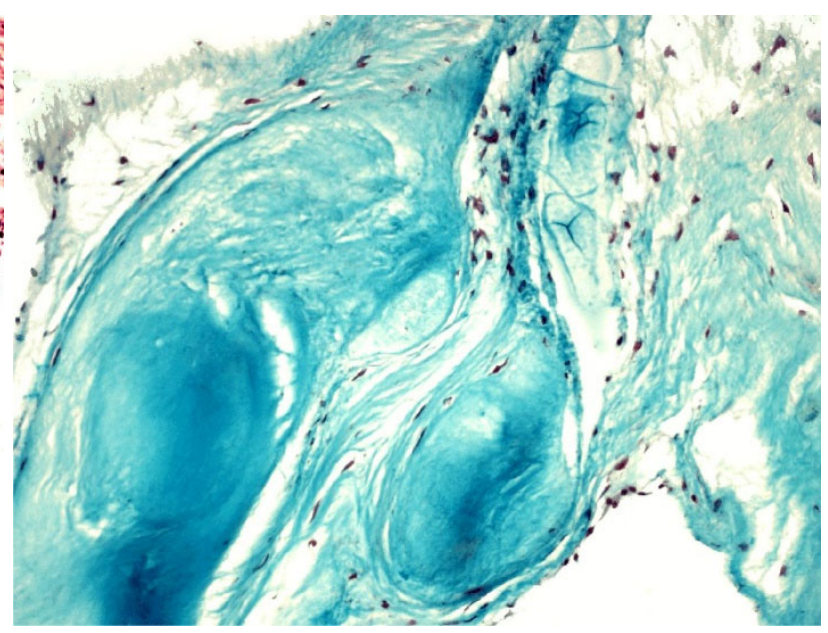

Figure 6 - Fibroelastoma. Dense avascular collagen in the central core of papillary structures, stained in greenish blue (Gömöri trichrome staining, ×40). 


\section{Case No. 2}

A 75-year-old woman presented in the Emergency Department with right-sided hemiparesis and an inferior altitudinal scotoma in the left eye, that developed over the last four hours. Her medical history included only arterial hypertension and she was on ACE blockers and statins. She was followed-up regularly by clinical exam echocardiography and her most recent check-up revealed normal functioning cardiac valves, moderate hypertrophy, and a normal ejection fraction. Upon arrival, a cerebral CT diagnosed no acute cerebral lesions. She was referred to our Department for further check-up. Transthoracic echography revealed a bulky cardiac tumor on the aortic side of the noncoronary aortic leaflet, near the left-noncoronary commissure. A transthoracic US exam was performed which showed, at the level of the aortic valve, the presence of a bulky, spheric, $12 \mathrm{~mm}$ tumor on the aortic aspect of the non-coronary aortic cusp (Figure 7). Her coronary and carotid angiography was normal. The patient had no fever or leukocytosis, and the endocarditis workup revealed no additional Duke criteria except her new-onset cardiac mass. She was referred to Department of Surgery for curative treatment, as the hemiparesis subsided gradually. The operation was performed by median sternotomy, under cardiopulmonary bypass. A transverse aortotomy gained access to the aortic valve, the inspection of which found a round, cauliflower- or anemone-like, white tumor, $10-12 \mathrm{~mm}$ in size, on the aortic aspect of the non-coronary leaflet, near the leftnon-coronary commissure (Figure 8). It was shaved and sent to Department of Pathology. The postoperative course was uneventful, and the patient was discharged on postoperative day 7 in sinus rhythm. The mass was fixed in $10 \%$ neutral buffered formalin, embedded in paraffin, and cut into sections. They were then stained with HE and Verhoeff's. Finally, immunohistochemistry was performed and was positive with monoclonal antibodies for the cluster of differentiation 31 (CD31) locus. The microscopic aspect was that of an avascular collagen core covered by a myxoid layer and numerous branch-like extensions (Figures 9 and 10). The ophthalmological examination (retinophotography and Fluorescein angiography) revealed two emboli at the superior temporal arterial branch, inducing its occlusion and retinal edema in the superior retinal sector (Figure 11, $\mathrm{A}$ and $\mathrm{B})$. The patient remains alive and well, two years after the surgical procedure.

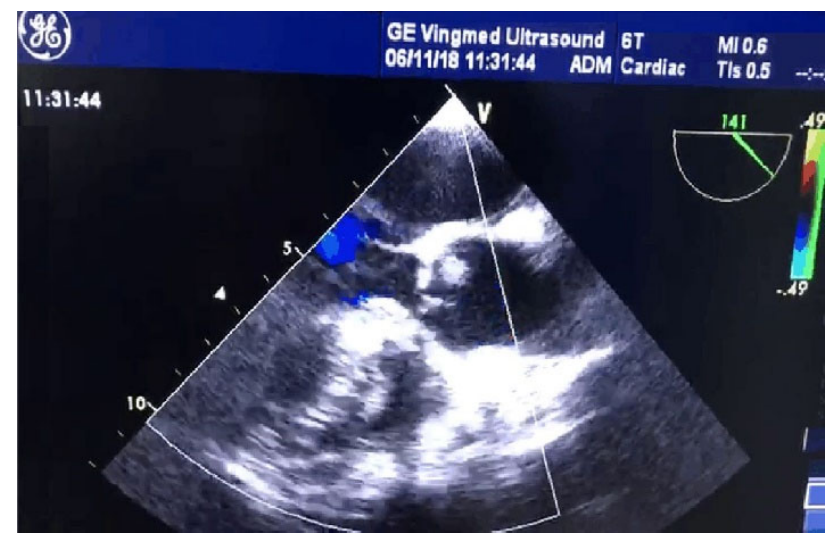

Figure 7 - Transthoracic long-axis view showing the presence of a large, round, mass on the aortic valve.



Figure 9 - Verhoeff's staining $(\times 40)$ of the specimen: collagen (red) and elastic fibers (black).

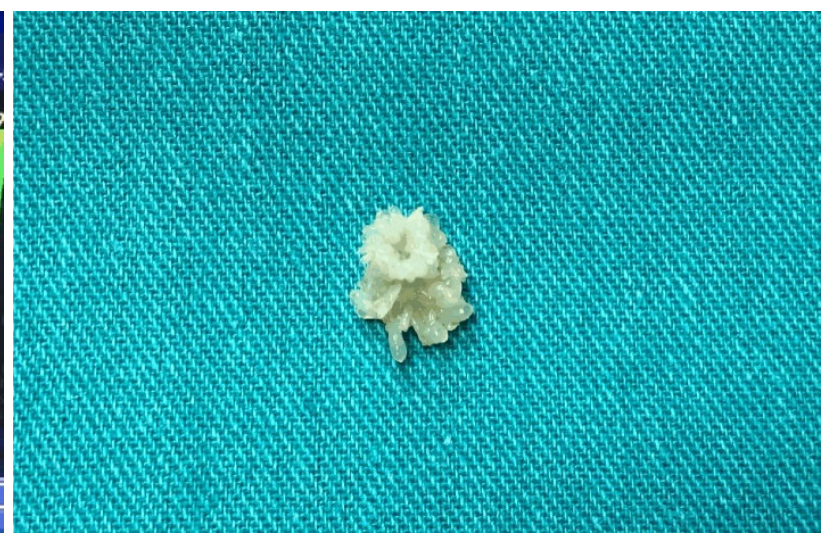

Figure 8 - The resected specimen: a cauliflower-like appearance with a central core and dendritic extensions.

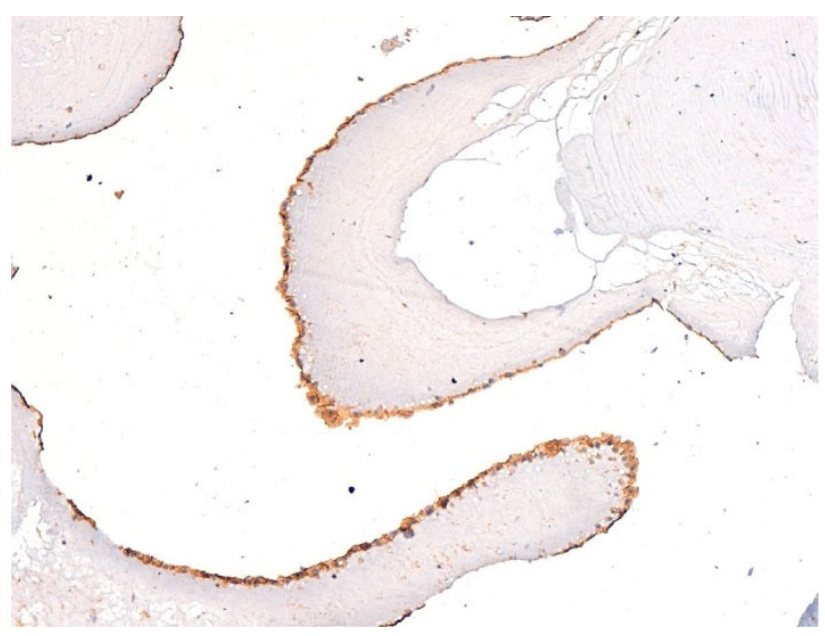

Figure 10 - Immunohistochemistry of the specimen by anti-CD31 monoclonal antibody immunostaining $(\times 40)$ revealing papillary structures covered by an endothelial lining. CD31: Cluster of differentiation 31. 

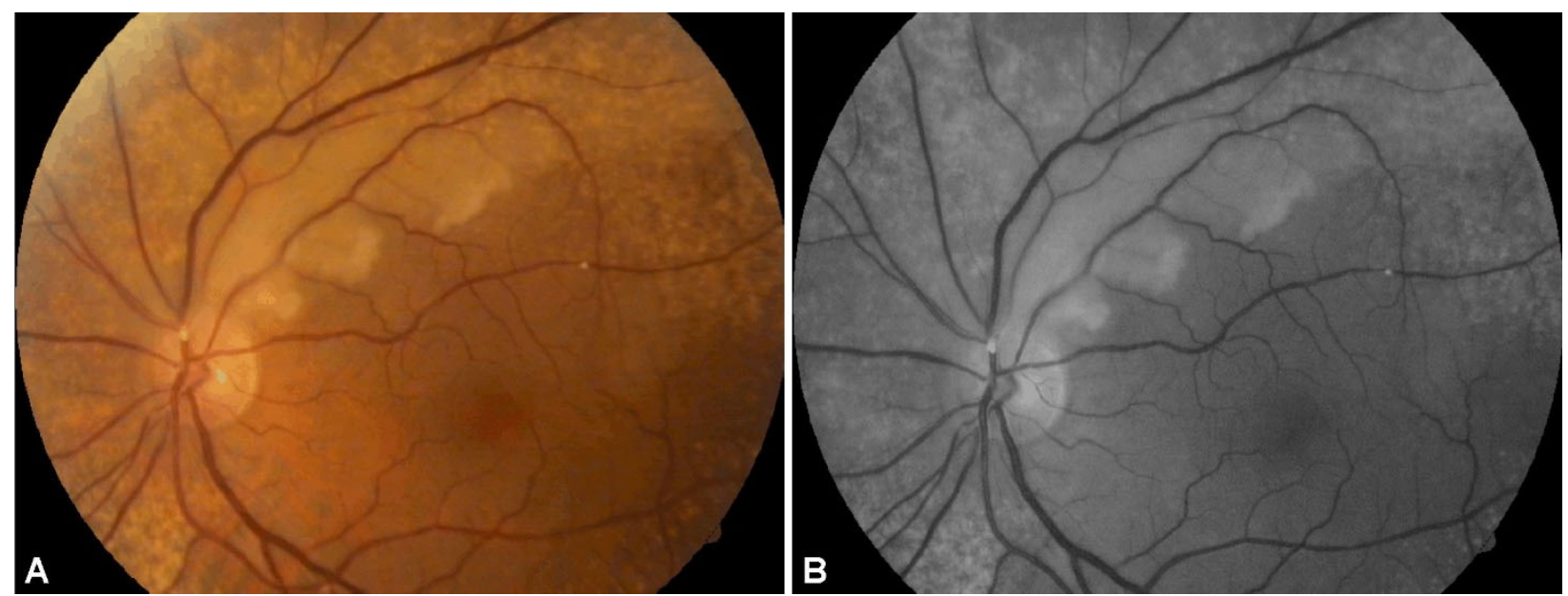

Figure 11 - (A) Color retinography at presentation: superior temporal branch arterial occlusion, retinal edema in the superior retinal sector, with two visible emboli. (B) Fluorescein angiography at presentation: delayed filling of the affected superior temporal artery by the Fluorescein, hypofluorescence in the surrounding area.

\section{口 Discussions}

CPF's are benign cardiac neoplasms. While traditionally they have been considered secondary to myxomas and lipomas, a recent report from the Mayo Group found their prevalence even higher than cardiac myxomas at a rate of 2:1 [2]. Most CPF's are isolated lesions found on valvular surfaces. Multiple lesions have been reported only in $7 \%$ of cases, but the number of concomitant lesions can be as high as 40 [9]. Their gross appearance is most frequently that of a sea anemone, but other aspects have also been described, particularly a finger-like lesion, or a gelatinous tumor $[11,12]$. Their clinical impact is due to their very high propensity for systemic embolization, particularly in the cerebral circulation, leading to symptoms of transient ischemic attacks (TIA) or CVAs. Far fewer are reports of embolizations in the coronary circulation $[6,7,13]$ or retinal artery $[14,15]$. Indeed, there are to the best of our knowledge only six reports in the medical literature of this uncommon association [14-19]. In a landmark report, which is to date the largest study on $\mathrm{CPF}$ and included 511 patients, of which 185 underwent surgical tumor removal, the Mayo Group found the rates of CVA's, TIA's and mortality increased in patients with echographic evidence of CPF who did not undergo surgery [2]. Close to $2 / 3$ of fibroelastomas are found on the aortic valve, while the mitral valve is affected in only $9 \%$ of cases [2]. In cases where the mitral valve is the location of the CPF, most are found on the anterior leaflet and the clinical signs can be those of heart failure due to mitral regurgitation, mostly by a ruptured primary chordae [20].

The etiology of CPF's is unknown. It has been noted that they are frequently associated with hypertrophic cardiomyopathy and previous cardiac surgery [21, 22]. The most interesting possibility has been raised by Grandmougin et al. who noted the presence of remnants of cytomegalovirus in the intermediate layer, suggesting the possibility of a virus-induced tumor [13].

Surgical resection has traditionally been performed using cardiopulmonary bypass via median sternotomy. Recently, minimal invasive techniques have been employed to that goal, including thoracoscopic $[23,24]$ or robotic access [25].

The first patient in our series presented the rare occurrence of multiple CPF's. Furthermore, the new onset of severe mitral regurgitation was due to the erosion of the free margin of the A2 segment due to the presence of the CPF's, as the patient had had a previous transesophageal exam, which found a normal functioning mitral valve. In our second case, the size of the aortic mass $(>1 \mathrm{~cm})$ in a patient with a previous CVA made surgery compulsory. Our patient was followed-up regularly for her hypertension and her most recent transthoracic US was normal, so the concomitant occurrence of the CVA, the branch retinal artery occlusion and the presence of a CPF at the level of the aortic valve was most likely due to embolization into the cerebral circulation. Regarding the ocular embolic complications, transluminal neodymium-doped yttrium aluminum garnet $(\mathrm{Nd}: \mathrm{YAG})$ laser embolysis procedure can be performed in order to achieve reperfusion of the occluded arterial branch [26]. Also, in cases with ocular involvement, beside the retinography and Fluorescein angiography, color Doppler US of the central retinal artery can be performed, in order to evaluate the severity of the arterial occlusion, and an associated giant cell arteritis must be excluded $[27,28]$.

\section{a Conclusions}

This report presents two cases of CPF's whose inaugural clinical expression was that of stroke or vision loss. The first case had multiple CPF's, while the second one had the rare association of retinal artery occlusion and CPF. These two rare cases underscore the risk posed by this neglected pathology, and we would argue that their mere presence warrants aggressive surgical resection due to the high risk of systemic embolization they carry.

\section{Conflict of interests}

The authors declare that they have no conflict of interests.

\section{References}

[1] Kumar V, Soni P, Hashmi A, Moskovits M. Aortic valve fibroelastoma: a rare cause of stroke. BMJ Case Rep, 2016, 2016:bcr2016217631. https://doi.org/10.1136/bcr-2016-217631 PMID: 27879304 PMCID: PMC5129025

[2] Tamin SS, Maleszewski JJ, Scott CG, Khan SK, Edwards WD, Bruce CJ, Oh JK, Pellikka PA, Klarich KW. Prognostic and 
bioepidemiologic implications of papillary fibroelastomas. J Am Coll Cardiol, 2015, 65(22):2420-2429. https://doi.org/10.1016/ j.jacc.2015.03.569 PMID: 26046736

[3] Grolla E, Dalla Vestra M, Zoffoli G, D'Ascoli R, Critelli A, Quatrale R, Mangino D, Rigo F. Papillary fibroelastoma, unusual cause of stroke in a young man: a case report. J Cardiothorac Surg, 2017, 12(1):33-35. https://doi.org/10.1186/s13019-0170592-6 PMID: 28526085 PMCID: PMC5437578

[4] Bevilacqua JA, Larraín E, Corredoira YA. Fibroelastoma of the mitral valve - a curable cause of stroke. Lancet Neurol, 2002 1(6):388-389. https://doi.org/10.1016/s1474-4422(02)00166-7 PMID: 12849402

[5] Burri H, Vuille C, Sierra J. Papillary fibroelastoma as a cause of cardioembolic stroke. Heart, 2002, 88(3):216. https://doi.org/ 10.1136/heart.88.3.216-a PMID: 12181206 PMCID: PMC 1767324

[6] Savage HO, Albanese A, Caruso V, Gedela S, Dungu J. Coronary embolization from aortic valve fibroelastoma. Clin Case Rep, 2020, 8(9):1610-1612. https://doi.org/10.1002/ ccr3.2954 PMID: 32983460 PMCID: PMC7495835

[7] Gonçalves M, Tralhão A, Trabulo M, Madeira M. Mitral valve papillary fibroelastoma as a cause of acute coronary syndrome. BMJ Case Rep, 2018, 11(1):bcr2018226930. https://doi.org/ 10.1136/bcr-2018-226930 PMID: 30567172 PMCID: PMC 6301527

[8] Neuman Y, Luthringer DJ, Kobal S, Miyamoto T, Trento A, Siegel RJ. Multiple aortic valve papillary fibroelastoma: an unusual presentation of a rare tumor. J Am Soc Echocardiogr 2003, 16(5):494-496. https://doi.org/10.1016/s0894-7317(03) 00117-2 PMID: 12724661

[9] Kumar TKS, Kuehl K, Reyes C, Talwar S, Moulick A, Jonas RA. Multiple papillary fibroelastomas of the heart. Ann Thorac Surg, 2009, 88(6):e66-e67. https://doi.org/10.1016/j.athoracsur.2009. 08.005 PMID: 19932221

[10] Diplaris K, Sidhom N, Berrebi A, Malergue MC, Lansac E. Multiple fibroelastoma: search and you will find. J Heart Valve Dis, 2015, 24(6):776-777. PMID: 27997785

[11] Sun JP, Asher CR, Yang XS, Cheng GG, Scalia GM, Massed AG Griffin BP, Ratliff NB, Stewart WJ, Thomas JD. Clinical and echocardiographic characteristics of papillary fibroelastomas: a retrospective and prospective study in 162 patients. Circulation, 2001, 103(22):2687-2693. https://doi.org/10.1161/01.cir.103. 22.2687 PMID: 11390338

[12] Nowacka A, Rancati V, Roumy A, Monney P, Marcucci CE, Kirsch M. Multiple cardiac papillary fibroelastomas - are they really rare or underdiagnosed? Heart Lung Circ, 2019, 28(4): e83-e85. https://doi.org/10.1016/j.hlc.2018.08.020 PMID: 30415831

[13] Grandmougin D, Delolme MC, Robin C, Vola M, Barral X. Incidental disclosure of asymptomatic coronary embolic occlusion related to mitral valve papillary fibroelastoma: an unusual finding and a review of the literature. J Heart Valve Dis, 2006, 15(1):136-139. PMID: 16480026

[14] Zamora RL, Adelberg DA, Berger AS, Huettner P, Kaplan HJ. Branch retinal artery occlusion caused by a mitral valve papillary fibroelastoma. Am J Ophthalmol, 1995, 119(3):325329. https://doi.org/10.1016/s0002-9394(14)71175-4 PMID: 7872394

[15] Shirani J, Bradlow JA, Metveyeva P, Losada M, Factor SM, Strom JA, Sisto D. Transient loss of vision as the presenting symptom of papillary fibroelastoma of aortic valve. Cardiovasc Pathol, 1997, 6(4):237-240. https://doi.org/10.1016/S10548807(96)00137-8 PMID: 25991541
[16] Lopez-Sanchez E, Muñoz EF, Aviño Martinez JA, Menezo Rozalen JL. Central retinal artery occlusion as the initial sign of aortic valve papillary fibroelastoma. Am J Ophthalmol, 2001, 131(5):667-669. https://doi.org/10.1016/s0002-9394(00)008 46-1 PMID: 11336952

[17] Ergun K, Golbasi Z, Cagli K, Altinsoy A, Mustafa Ulas M, Temucin T, Arat N, Topaloglu S, Deveci B, Ertan A. A rare cause of sudden vision loss in a young male: papillary fibroelastoma of the aortic valve. Echocardiography, 2007, 24(4):425-429. https://doi.org/10.1111/j.1540-8175.2006.00 410.x PMID: 17381654

[18] Martín M, Benito EM, Corros C, González M. [Central retinal artery thrombosis and mitral papillary fibroelastoma]. Med Clin (Barc), 2012, 138(14):642. https://doi.org/10.1016/j.med cli.2010.09.046 PMID: 21295798

[19] Henao M, Chamchikh J, Chalam KV. Sudden vision loss from central retinal artery occlusion as a presenting symptom of mitral valve papillary fibroelastoma. Retin Cases Brief Rep, 2019 Sep 30. https://doi.org/10.1097//CB.0000000000000915 PMID: 31574007

[20] Prifti E, Ikonomi M, Veshti A, Demiraj A, Xhaxho R. Papillary fibroelastoma of the anterior leaflet of the mitral valve mimicking vegetation. Int J Surg Case Rep, 2015, 14:19-22. https://doi.org/10.1016/j.ijscr.2015.07.003 PMID: 26209756 PMCID: PMC4573212

[21] Carrigan TP, Galla JM, Smedira NG, Stewart WJ. Late diagnosis of multiple fibroelastomas in a 41-year-old woman with hypertrophic cardiomyopathy and atrial fibrillation. J Am Soc Echocardiogr, 2008, 21(12):1391.e1-1391.e3. https:// doi.org/10.1016/j.echo.2008.09.019 PMID: 19041583

[22] Kurup AN, Tazelaar HD, Edwards WD, Burke AP, Virmani R, Klarich KW, Orszulak TA. latrogenic cardiac papillary fibroelastoma: a study of 12 cases (1990 to 2000). Hum Pathol, 2002, 33(12):1165-1169. https://doi.org/10.1053/hupa.2002. 130105 PMID: 12514783

[23] Fatehi Hassanabad A, El Idrissi KR, Kent WDT. Right anterior minithoracotomy approach for resection of papillary fibroelastoma. J Card Surg, 2020, 35(7):1729-1731. https://doi.org/10.1111/ jocs.14674 PMID: 32485064

[24] Topal B, Hindori V, Pouwels S, Riezebos R. The right anterior thoracotomy approach to resect a cardiac papillary fibroelastoma of the aortic valve. Cureus, 2020, 12(2):e7136. https://doi.org/10.7759/cureus.7136 PMID: 32257681 PMCID: PMC7105234

[25] Nisivaco SM, Patel B, Balkhy HH. Robotic totally endoscopic excision of aortic valve papillary fibroelastoma: the least invasive approach. J Card Surg, 2019, 34(12):1492-1497. https://doi.org/10.1111/jocs.14291 PMID: 31609505

[26] Stanca HT, Petrović Z, Munteanu M. Transluminal Nd:YAG laser embolysis - a reasonable method to reperfuse occluded branch retinal arteries. Vojnosanit Pregl, 2014, 71(11):10721077. https://doi.org/10.2298/vsp130511056s PMID: 25536813

[27] Jianu DC, Jianu SN, Munteanu M, Vlad D, Rosca C, Petrica L. Color Doppler imaging features in patients presenting central retinal artery occlusion with and without giant cell arteritis. Vojnosanit Pregl, 2016, 73(4):397-401. https://doi.org/10.22 98/VSP140814087C PMID: 29309110

[28] Stanca HT, Suvac E, Munteanu M, Jianu DC, Motoc AGM, Roşca GC, Boruga O. Giant cell arteritis with arteritic anterior ischemic optic neuropathy. Rom J Morphol Embryol, 2017, 58(1):281-285. PMID: 28523333

\section{Corresponding authors}

Marius Cristian Şuţă, MD, PhD Student, Department of Ophthalmology, Victor Babeş University of Medicine and Pharmacy, 2 Eftimie Murgu Square, 300041 Timişoara, Timiş County, Romania; Phone +40726-848 616, e-mail: sutamarius@yahoo.ro

Diana-Camelia Bonţe, MD, PhD, Department of Biochemistry, Victor Babeş University of Medicine and Pharmacy, 2 Eftimie Murgu Square, 300041 Timişoara, Timiş County, Romania; Phone +40724-017 080, e-mail: dia_bonte@yahoo.com 